hypothesis, $|\xi|=1$, of the inequalities (8) is satisfied by the vector $\xi=e(t)$, it now follows from (8) that

$$
\lambda(t) \leq d \log r(t) / d t \leq \mu(t) .
$$

Finally, if $t^{0}<u<v$, integration of (9) between $t=u$ and $t=v$ gives

$$
\int_{u}^{v} \lambda(t) d t \leq \log r(v)-\log r(u) \leq \int_{u}^{0} \mu(t) d t .
$$

On the other hand, the convergence of the improper integrals (6) means that

$$
\int_{u}^{0} \lambda(t) d t \rightarrow 0 \text { and } \int_{u}^{v} \mu(t) d t \rightarrow 0 \text { if } u \rightarrow \infty, v \rightarrow \infty .
$$

But the last two formula lines imply that $\log r(v)-\log r(u) \rightarrow 0$ as $u \rightarrow \infty, v \rightarrow \infty$. This means that the logarithm of $r(t)$ tends to a finite limit as $t \rightarrow \infty$. Since this is equvalent to the statement that $r(t)$ itself tends to a finite non-vanishing limit, the proof is complete.

\title{
A GENERALIZATION OF ALFREY'S THEOREM FOR VISCO-ELASTIC MEDIA*
}

\section{By H. S. TSIEN (California Institute of Technology)}

1. Introduction. For the non-homogeneous stresses in isotropic incompressible viscoelastic media characterized by linear relations between the components of stress, strain and their derivatives with respect to time, T. Alfrey has shown (Ref. 1) that in the case of the first boundary value problem, the stress distribution is identical with that in an incompressible elastic material under the same instantaneous surface forces. A similar result was obtained for the second boundary value problem where the displacements at the boundary are specified. It is the purpose of the present note to generalize this theorem to isotropic compressible media for problems involving body forces. Only the first boundary value problem will be discussed, as the corresponding theorem on the second boundary value problem is self-evident.

2. First boundary value problem. Let the displacements along the $x, y, z$ directions be $u, v, w$. Then the typical expressions** of the six strain components can be written as:

$$
\begin{aligned}
\epsilon_{x} & =\frac{\partial u}{\partial x}, \\
\gamma_{x y} & =\frac{\partial u}{\partial y}+\frac{\partial v}{\partial x} .
\end{aligned}
$$

If the six stress components are denoted by $\sigma_{x}, \sigma_{y}, \sigma_{z}, \tau_{x y}, \tau_{y z}, \tau_{z x}$, the components

*Received Sept. 7, 1949.

**Throughout this note, only typical expressions are explicitly given; other expressions can be readily obtained by cyclic permutations. 
of body force by $X, Y, Z$, and the surface force per unit area by $\bar{X}, \bar{Y}, \bar{Z}$, the equations of equilibrium are

$$
\frac{\partial \sigma_{x}}{\partial x}+\frac{\partial \tau_{x y}}{\partial y}+\frac{\partial \tau_{z x}}{\partial z}+X=0
$$

Here the body forces $X, Y, Z$ are the result of external field or agent and will not be identified with the inertia forces of the material. The inertia forces are here considered to be negligible as is actually the case for a wide class of problems. If $l, m, n$ are the direction cosines of the normal to the surface, then the surface conditions are:

$$
\bar{X}=l \sigma_{x}+m \tau_{x y}+n \tau_{z x}
$$

To determine the stress distribution completely, there are in addition six equations of compatibility:

$$
\frac{\partial^{2} \epsilon_{x}}{\partial y^{2}}+\frac{\partial^{2} \epsilon_{y}}{\partial x^{2}}=\frac{\partial^{2} \gamma_{x y}}{\partial x \partial y}, \quad 2 \frac{\partial^{2} \epsilon_{x}}{\partial y \partial z}=\frac{\partial}{\partial x}\left(-\frac{\partial \gamma_{\nu z}}{\partial x}+\frac{\partial \gamma_{z x}}{\partial y}+\frac{\partial \gamma_{x y}}{\partial z}\right)
$$

It remains to specify the relations between the components of stress, strain and their time derivatives. These relations will be assumed to be linear, corresponding to problems of small strain. If in addition the material is assumed to be isotropic, then purely on the ground of invariance under space coordinate transformation it can be shown that the required relations have to be of the following form:

$$
\begin{aligned}
\mathbf{P} \sigma_{x} & =\mathbf{Q}\left(\lambda e+2 \mu \epsilon_{x}\right) \\
\mathbf{P} \tau_{x y} & =\mathbf{Q} \mu \gamma_{x \nu},
\end{aligned}
$$

where $\mu$ and $\lambda$ are constants, and

$$
e=\epsilon_{x}+\epsilon_{y}+\epsilon_{z}
$$

The operators $\mathbf{P}$ and $\mathbf{Q}$ are time operators defined as:

$$
\begin{aligned}
& \mathbf{P}=\frac{\partial^{m}}{\partial t^{m}}+a_{m-1} \frac{\partial^{m-1}}{\partial t^{m-1}}+\cdots+a_{0} \\
& \mathbf{Q}=\frac{\partial^{n}}{\partial t^{n}}+b_{n-1} \frac{\partial^{n-1}}{\partial t^{n-1}}+\cdots+b_{0}
\end{aligned}
$$

The $a$ 's and $b$ 's define the characteristics of the material. They could be functions of time, but not functions of the space variables. Thus a material with changing properties, caused by the drift towards thermodynamic and chemical equilibrium, can be also represented by these operators.

By eliminating the strains between the compatibility equations (4) and the stressstrain relations (5), one has:

$$
\begin{array}{r}
\mathbf{P}\left[\nabla^{2} \sigma_{x}+\frac{2 \lambda+2 \mu}{3 \lambda+2 \mu} \frac{\partial^{2} \Theta}{\partial x^{2}}+\frac{\lambda}{\lambda+2 \mu}\left(\frac{\partial X}{\partial x}+\frac{\partial Y}{\partial y}+\frac{\partial Z}{\partial z}\right)+2 \frac{\partial X}{\partial x}\right]=0 \\
\mathbf{P}\left[\nabla^{2} \tau_{x y}+\frac{2 \lambda+2 \mu}{3 \lambda+2 \mu} \frac{\partial^{2} \Theta}{\partial x \partial y}+\left(\frac{\partial X}{\partial y}+\frac{\partial Y}{\partial x}\right)\right]=0
\end{array}
$$


where

$$
\Theta=\sigma_{x}+\sigma_{\nu}+\sigma_{z}
$$

The equations (8) are sufficient to solve the first boundary value problem: The surface forces $\bar{X}, \bar{Y}, \bar{Z}$ and body forces $X, Y, Z$ are specified for all values of $t$. For any given $t$, these forces must be in equilibrium. The problem is to determine the distribution of stresses fulfilling these boundary conditions.

Now let

$$
\bar{X}=\bar{X}^{*} g(t), \quad \bar{Y}=\bar{Y}^{*} g(t), \quad \bar{Z}=\bar{Z}^{*} g(t)
$$

The starred quantities are functions of space coordinates only. Then for equilibrium the body force must vary with time in a similar way. Thus:

$$
X=X^{*} g(t), \quad Y=Y^{*} g(t), \quad Z=Z^{*} g(t)
$$

The stress components can now be written in the same manner:

$$
\sigma_{x}=\sigma_{x}^{*} g(t), \quad \tau_{x y}=\tau_{x y}^{*} g(t),
$$

By substituting equations (11) and (12) into equations (8), it is easily shown that the starred quantities satisfy the stress equations for a purely elastic medium with Lamés constants $\lambda$ and $\mu$. By substituting equations (10) and (12) into the boundary conditions (3), it is seen that the starred quantities also satisfy their corresponding boundary conditions. Therefore, in the case of the first boundary value problem, the stress distribution is identical with that in purely elastic material under the same instantaneous surface forces and body forces.

To determine the displacements $u, v$ and $w$, one introduces the unknown time function $h(t)$ such that:

$$
u=u^{*} h(t), \quad v=v^{*} h(t), \quad w=w^{*} h(t)
$$

where the starred quantities are again functions of space variables only. When equations (13) are substituted into equations (5), $u^{*}, v^{*}, w^{*}$ are found to be the displacements of a purely elastic medium under the loading $\bar{X}^{*}, \bar{Y}^{*}, \bar{Z}^{*}$ and $X^{*}, Y^{*}, Z^{*}$. Furthermore, $h(t)$ is determined by:

$$
\mathbf{Q} h(t)=\mathbf{P} g(t)
$$

with the initial condition that at $t=0, h$ and its first $(n-1)$ derivatives vanish. The function $h(t)$ is thus universal in the sense that it depends only on $g(t)$ and the properties of the material. The other characteristics of the problem do not enter into its determination. In particular, the function $h(t)$ may be directly determined experimentally on a pure tension bar with the tension varied with time according to $g(t)$.

By superimposing solutions, the time dependence of the applied forces can be generalized as shown by Alfrey (Ref. 1). 